$41-53$

\title{
Surgical versus injection treatment for injection-confirmed chronic sacroiliac joint pain
}

Authors William Ryan Spiker ${ }^{1}$, Brandon D Lawrence ${ }^{1}$, Annie L Raich ${ }^{2}$, Andrea C Skelly², Darrel S Brodke ${ }^{1}$

Institutions ${ }^{1}$ Department of Orthopaedic Surgery, University of Utah, Salt Lake City, UT, USA

${ }^{2}$ Spectrum Research Inc, Tacoma, WA, USA

\section{ABSTRACT}

Study design: Systematic review.

Study rationale: Chronic sacroiliac joint pain (CSJP) is a common clinical entity with highly controversial treatment options. A recent systematic review compared surgery with denervation, but the current systematic review compares outcomes of surgical intervention with therapeutic injection for the treatment of CSJP and serves as the next step for evaluating current evidence on the comparative effectiveness of treatments for non-traumatic sacroiliac joint pain.

Objective or clinical question: In adult patients with injection-confirmed CSJP, does surgical treatment lead to better outcomes and fewer complications than injection therapy?

Methods: A systematic review of the English-language literature was undertaken for articles published between 1970 and June 2012. Electronic databases and reference lists of key articles were searched to identify studies evaluating surgery or injection treatment for injection-confirmed CSJP. Studies involving traumatic onset or non-injection-confirmed CSJP were excluded. Two independent reviewers assessed the level of evidence quality using the grading of recommendations assessment, development and evaluation (GRADE) system, and disagreements were resolved by consensus.

Results: We identified twelve articles (seven surgical and five injection treatment) meeting our inclusion criteria. Regardless of the type of treatment, most studies reported over $40 \%$ improvement in pain as measured by Visual Analog Scale or Numeric rating Scale score. Regardless of the type of treatment, most studies reported over $20 \%$ improvement in functionality. Most complications were reported in the surgical studies.

Conclusion: Surgical fusion and therapeutic injections can likely provide pain relief, improve quality of life, and improve work status. The comparative effectiveness of these interventions cannot be evaluated with the current literature. 


\section{STUDY RATIONALE AND CONTEXT}

The sacroiliac joint is a common source of low back pain as well as referred pain into the buttock, groin, and lower extremities. Degeneration of the sacroiliac joint can be caused by numerous conditions, including degenerative and inflammatory arthritis, trauma, rheumatological conditions, pregnancy, lumbosacral fusion, hip arthritis, limb length inequality, infections, and neoplasia. Although sacroiliac joint pathology has historically been difficult to diagnose based on clinical signs and symptoms, recent studies have solidified pain relief with image intensifier-guided injection of a local anesthetic as the gold standard for diagnosis. However, treatment of chronic sacroiliac joint pain (CSJP) remains highly controversial. Treatment options include oral antiinflammatory medications, physical therapy, steroid injections, radiofrequency ablation, and surgical fusion. A previous review comparing surgery and denervation suggested that improvements in pain, patient satisfaction, and functional outcomes were similar based on indirect comparison of case series [1]. In this systematic review, we compare the effectiveness of intraarticular steroid injections to surgical intervention on the clinically relevant outcomes of pain relief, quality of life, and return to work.

\section{OBJECTIVE OR CLINICAL QUESTION}

In patients 18 years or older with injection-confirmed CSJP, does surgical treatment lead to better outcomes and fewer complications than injection therapy?

\section{MATERIALS AND METHODS}

Study design: Systematic review.

Search: PubMed; Cochrane and National Guideline Clearinghouse Databases; bibliographies of key articles.

Dates searched: 1970 through June 7, 2012.

Inclusion criteria: Studies of surgical or injection treatment in patients 18 years or older with injectionconfirmed CSJP.

Exclusion criteria: Studies in patients younger than 18 years; those with previous sacroiliac joint surgery on the same side; trauma; neoplasms; iatrogenic conditions; infection; pain from pregnancy; metabolic disorders; inflammatory disease; studies of fewer than nine patients; nonhuman in vivo, in vitro, and biomechanical studies.

Outcomes: Pain (Visual Analog Scale [VAS], Numeric Rating Scale [NRS]); functionality (Oswestry Disability Index [ODI]), Majeed Score, SF-36 (ShortForm 36); AAOS/MODEMS (American Academy/ Association of Orthopaedic Surgeons/Musculoskeletal Outcomes Data Evaluation and Management System); fusion rate; return to work; complication rate.

Analysis: Descriptive statistics, means, standard deviation, and ranges were abstracted from the original reports as available. Mean percentage improvement in outcome scores was calculated by dividing the change score from baseline to follow-up by the baseline score to get the total percentage improvement. Pooling of data was not done due to concerns regarding study quality and heterogeneity of treatments and study populations.

Details about methods can be found in the electronic supplemental Web Appendix at www.aospine.org/ebsj 


\section{RESULTS}

- From a total of 303 citations retrieved, 32 were evaluated for full-text review, and twelve met the inclusion criteria (Fig 1). A diagnosis of sacroiliac joint pain was confirmed by injection in all studies.

- Seven case series (CoE IV) evaluated surgery (fusion or debridement) for chronic sacloiliac joint pain [2-8]. Five studies evaluated injection therapy for sacroiliac joint pain using corticosteroid, Botulinum toxin, or prolotherapy [9-13]. Three of the injection studies were comparative studies; however, these are considered case series (CoE IV) for this review, with each treatment arm considered separately $[9,11,12]$.

- Since no studies comparing surgery with injection therapy in the same population were identified, conclusions from direct treatment comparisons are not possible. Populations in included studies were predominantly female and middle-aged (Table 1).

Further details on the class of evidence rating for these studies can be found in the supplemental Web Appendix at www.aospine.org/ebsj.
Fig 1 Results of literature search.
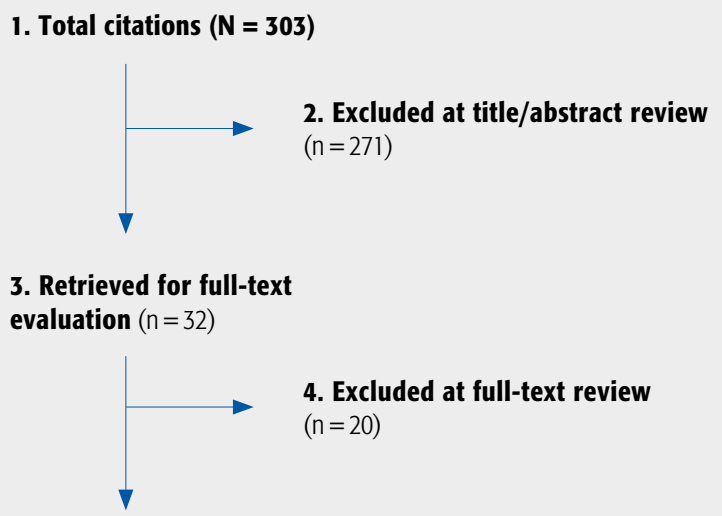

5. Included publications $(n=12)$

\begin{tabular}{|c|c|c|c|c|}
\hline $\begin{array}{l}\text { Author } \\
\text { (Year) }\end{array}$ & $\begin{array}{l}\text { Demographics }{ }^{\dagger} / \\
\text { follow-up }\end{array}$ & $\begin{array}{l}\text { Patient } \\
\text { characteristics }\end{array}$ & $\begin{array}{l}\text { Requirement(s) for confirmed } \\
\text { diagnosis }\end{array}$ & Intervention(s) \\
\hline \multicolumn{5}{|c|}{ Surgical studies } \\
\hline 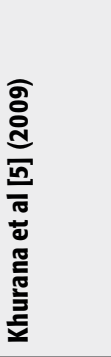 & $\begin{array}{l}\mathrm{N}=15(19 \text { joints) } \\
\text { Age: } 48.7(37.3-62.6) \text { y } \\
\text { Male: } 26.7 \% \text {, unilateral }(n=11) \\
\text { Bilateral }(n=4) \\
\text { Duration of pain: NR (chronic nontraumatic } \\
\text { condition) } \\
\text { FU: mean } 17 \text { (range, 9-39) mo (\% NR) }\end{array}$ & $\begin{array}{l}\text { Principal symptom: low } \\
\text { back pain or pain in } \\
\text { buttock: } \\
\text { - OA, } n=7 \\
\text { - SIJ dysfunction, } n=4 \\
\text { - SIJ instability, } n=3 \\
\text { - Inflammatory arthritis, } \\
n=1 \\
\text { Previous spinal surgery, } \\
n=6\end{array}$ & $\begin{array}{l}\text { All the following: } \\
\text { - Image intensifier-guided single injection of local } \\
\text { anesthetic + corticosteroid with resulting pain } \\
\text { relief, time period and \% pain relief NR } \\
\text { - Clinical evaluation: Patrick test, Gaenslen test, } \\
\text { tenderness over posterior SIJ } \\
\text { - Radiographic evaluation: plain radiographs, CT, } \\
\text { MRI }\end{array}$ & $\begin{array}{l}\text { Percutaneous sacroiliac } \\
\text { fusion using hollow } \\
\text { modular anchorage screws } \\
\text { packed with demineralized } \\
\text { bone matrix (DBX) }\end{array}$ \\
\hline 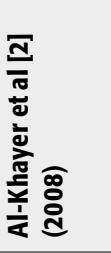 & $\begin{array}{l}N=9(12 \text { joints }) \\
\text { Age: } 42.4 \pm 6.5 \text { (range, } 35-56) \text { y } \\
\text { Male: } 0 \% \text {, unilateral }(n=6) \\
\text { Bilateral }(n=3) \\
\text { Duration of symptoms: } 30 \pm 21(12-84) \text { mo } \\
\text { FU: mean } 40 \pm 15.4(24-70) \text { mo ( } \% \text { NR) }\end{array}$ & $\begin{array}{l}\text { Chronic SIJ pain with } \\
\text { pain and tenderness over } \\
\text { sacral sulcus and } \\
\text { posterior SIJ }\end{array}$ & $\begin{array}{l}\text { Image intensifier-guided SIJ block with temporary } \\
\text { pain relief; injectant, time period, and \% pain } \\
\text { relief NR } \\
\text { Additional evaluations: } \\
\text { - Clinical: Patrick (Faber) test } \\
\text { - Radiographic: plain radiographs }\end{array}$ & $\begin{array}{l}\text { Percutaneous sacroiliac } \\
\text { joint arthrodesis using } \\
\text { hollow modular anchorage } \\
\text { screws filled with } \\
\text { demineralized bone matrix } \\
\text { mixed with bone reaming } \\
\text { from surgical procedure }\end{array}$ \\
\hline 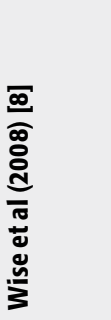 & $\begin{array}{l}\mathrm{N}=13 \text { (19 joints) } \\
\text { Age: } 53.1 \text { (range, } 45-62) \text { y } \\
\text { Male: } 7.7 \% \text {, bilateral }(n=6) \\
\text { Unilateral ( } n=7) \\
\text { Duration of pain: NR (patients had received } \\
\text { conservative treatment for } 6 \text { mo to several } \\
\text { years) } \\
\text { FU: mean } 29.5 \text { (range } 24-35) \text { mo (\% NR) }\end{array}$ & $\begin{array}{l}\text { SIJ dysfunction (lumbar } \\
\text { region excluded as pain } \\
\text { region) } \\
\text { Previous spine surgery: } \\
\text { - Lumbar fusion } \\
\text { extending to sacrum, } \\
n=8 \\
\text { - Open SI fusion on } \\
\text { contralateral side, } n=1\end{array}$ & $\begin{array}{l}\text { Image intensifier-guided single intraarticular } \\
\text { injection of lidocaine + triamcinolone with pain } \\
\text { relief of } 75 \% \text { on VAS scale within } 30 \text { min and } \\
\text { lasting } \geq 2 \mathrm{~h} \\
\text { Additional evaluations: } \\
\text { - Clinical: physical examination, details NR; pain } \\
\text { referral patterns, details NR }\end{array}$ & $\begin{array}{l}\text { Percutaneous fusion via } \\
\text { posterior approach in } \\
\text { longitudinal axis of SIJ } \\
\text { using threaded cage with } \\
\text { BMP (INFUSE BMP2, } \\
\text { off-label use) }\end{array}$ \\
\hline
\end{tabular}




\begin{tabular}{|c|c|c|c|c|}
\hline $\begin{array}{l}\text { Author } \\
\text { (Year) }\end{array}$ & $\begin{array}{l}\text { Demographics }{ }^{\dagger} / \\
\text { follow-up }\end{array}$ & $\begin{array}{l}\text { Patient } \\
\text { characteristics }\end{array}$ & $\begin{array}{l}\text { Requirement(s) for confirmed } \\
\text { diagnosis }\end{array}$ & Intervention(s) \\
\hline 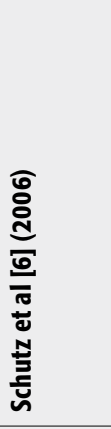 & $\begin{array}{l}\mathrm{N}=17 \text { (34 joints) } \\
\text { Age: } 43.2(22-76) \text { y } \\
\text { Male: } 29.4 \% \\
\text { Bilateral }(n=17) \\
\text { Duration of pain: } 6.6 \text { (range, 1-20) y } \\
\text { FU: Mean } 39 \text { (range, 12-66) mo (\% NR) }\end{array}$ & $\begin{array}{l}\text { Chronic SIJ syndrome: } \\
\text { - Posttraumatic, } n=5 \\
\text { - Idiopathic SIJ } \\
\text { degeneration, } n=12 \\
\text { - Previous spine } \\
\text { surgery: } \\
\text { - Lumbar, } n=10 \\
\text { - SIJ, } n=1\end{array}$ & $\begin{array}{l}\text { One or more of the following: } \\
\text { - Image intensifier-guided single injection of } \\
\text { scandicaine with temporary reduction of pain } \\
\text { - Clinical evaluation: Mennell sign } \\
\text { (hyperextension test), pain provocation by SIJ } \\
\text { compression } \\
\text { - Radiographic: Barsony technique; CT scan; } \\
\text { Technetium scan } \\
\text { - Psychological, social, occupational: details NR } \\
\text { - Selective immobilization of lumbar, } \\
\text { lumbosacral, SI segments with temporary } \\
\text { external fixator }\end{array}$ & $\begin{array}{l}\text { Fusion with internal } \\
\text { fixation and decortication } \\
\text { of SIJ using separate } \\
\text { approach to each joint } \\
\text { (modified Verral and Pitkin } \\
\text { dorsal bilateral } \\
\text { interlocking technique); } \\
\text { iliac crest autograft } \\
\text { performed. }\end{array}$ \\
\hline 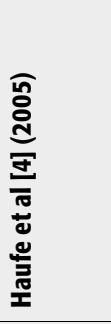 & $\begin{array}{l}\mathrm{N}=38 \text { (No. of joints NR) } \\
\text { Age: } 66 \text { ( } 43-81 \text {, median } 68) \text { y; male: } 46 \% \\
\text { Bilateral NR, unilateral NR } \\
\text { Duration of pain: } 10.4 \text { (median, } 7.5) \text { y } \\
\text { FU: } \geq 2 \text { y ( } \% \text { NR) }\end{array}$ & NR & $\begin{array}{l}\text { Image intensifier-guided single injection of } \\
\text { bupivacaine in posterior superior iliac crest tendon } \\
\text { with } \geq 75 \% \text { reduction in pain for } \geq 1 \mathrm{~h}\end{array}$ & $\begin{array}{l}\text { Debridement: } \\
\text { electrocautery and } \\
\text { holmium laser to denude } \\
\text { bone of ligamental } \\
\text { insertions on ilium crest } \\
\text { and remove capsular/ } \\
\text { nervous tissues of joint; } \\
\text { hand-burr used to smooth } \\
\text { iliac surface }\end{array}$ \\
\hline 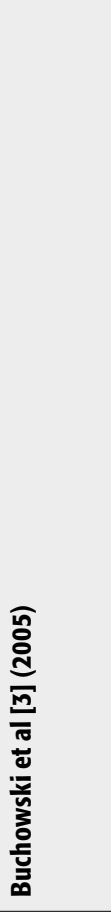 & $\begin{array}{l}\mathrm{N}=20 \text { (No. of joints NR) } \\
\text { Age: } 45.1 \pm 12.7 \mathrm{y} \\
\text { Male: } 15 \% \\
\text { Unilateral NR } \\
\text { Bilateral NR } \\
\text { Duration of symptoms: } 2.6 \pm 1.9 \mathrm{y} \\
\text { FU: mean } 5.8 \pm 1.9 \text { y (100\% for radiographic } \\
\text { outcome, } 75 \% \text { for functional outcome) }\end{array}$ & $\begin{array}{l}\text { Low back, buttock, and/ } \\
\text { or leg pain: } \\
\text { - SIJ dysfunction, } n=13 \\
\text { - SIJ OA, } n=5 \\
\text { - SIJ IA, } n=1 \\
\text { - SIJ postpartum } \\
\text { instability, } \\
n=1 \\
\text { Previous spine surgery } \\
\text { (overlapping categories): } \\
\text { - Fusion to sacrum, } n=8 \\
\text { - ICBG, } n=10 ; 9 \text { of them } \\
\text { developed ipsilateral } \\
\text { SIJ symptoms } \\
\text { - Any spine surgery, } \\
n=15 ; 3.5 \pm 3.7 \\
\text { surgeries/patient } \\
\text { (range, } 1-13 \text { ) } \\
\text { - Failed traditional, } \\
\text { nonoperative } \\
\text { treatment, } \\
n=20 \\
\text { - Comorbidities } \\
\text { including } \\
\text { hypertension, asthma, } \\
\text { CAD, } n=10\end{array}$ & $\begin{array}{l}\text { Image intensifier-guided intraarticular injections } \\
\text { (mean, 2.7; range, 2-4) of local anesthetic/ } \\
\text { glucocorticosteroid with recurrence of symptoms } \\
\text { after initial positive response, time period and \% } \\
\text { relief NR } \\
\text { Additional evaluations: } \\
\text { - Clinical: Patrick test, Gaenslen test, palpitation } \\
\text { over SIJ, compression test, hip abduction test } \\
\text { - Radiographic: plain radiographs; triple-phase } \\
\text { bone scan, MRI, CT }\end{array}$ & $\begin{array}{l}\text { SIJ arthrodesis using } \\
\text { modified Smith-Petersen } \\
\text { technique }\end{array}$ \\
\hline 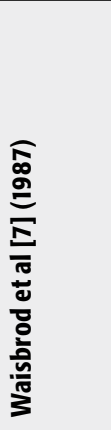 & $\begin{array}{l}\mathrm{N}=21(22 \text { joints }) \\
\text { Age: } 42(20-58) \text { y } \\
\text { Male: } 14.3 \% \\
\text { Bilateral }(n=1) \\
\text { Unilateral }(n=20) \\
\text { Duration of pain: }>2 \text { y } \\
\text { FU: mean } 30 \text { (range, } 12-55) \text { mo (\% NR) }\end{array}$ & $\begin{array}{l}\text { Overt OA (pain localized } \\
\text { in } \mathrm{SI} \text { area and lower back } \\
\text { with varying } \\
\text { radiculopathy) } \\
\text { Previous spine surgery: } \\
\text { - Discectomy, } \mathrm{n}=2 \\
\text { - Posterolateral fusion, } \\
\mathrm{n}=5 \\
\text { - Bilateral total hip } \\
\quad \text { replacement, } \mathrm{n}=2 \\
\text { - Chiari pelvic } \\
\text { osteotomy, } \mathrm{n}=2\end{array}$ & $\begin{array}{l}\text { Positive response for all the following: } \\
\text { - Provocation test: 10\% NaCl solution to } \\
\text { reproduce pain pattern } \\
\text { - Single injection of local anesthetic "under } \\
\text { television control" to relieve pain completely for } \\
\text { duration of drug's effect } \\
\text { - Clinical evaluation: Patrick test, Gaenslen test, } \\
\text { tenderness in SIJ area } \\
\text { - Psychological evaluation: FAPK, MMPI, FPI } \\
\text { - Radiography: scintigraphy (technetium), plain } \\
\text { radiographs, CT }\end{array}$ & $\begin{array}{l}\text { SIJ arthrodesis: articular } \\
\text { surfaces completely } \\
\text { excised; corticocancellous } \\
\text { bone graft taken from iliac } \\
\text { crest and/or } \\
\text { tricalciumphosphate } \\
\text { ceramic blocks interposed } \\
\text { under pressure between } \\
\text { denuded cancellous bone }\end{array}$ \\
\hline
\end{tabular}


Table 1 (Cont.)

\begin{tabular}{|c|c|c|c|c|}
\hline $\begin{array}{l}\text { Author } \\
\text { (Year) }\end{array}$ & $\begin{array}{l}\text { Demographics }{ }^{\dagger} / \\
\text { follow-up }\end{array}$ & $\begin{array}{l}\text { Patient } \\
\text { characteristics }\end{array}$ & $\begin{array}{l}\text { Requirement(s) for confirmed } \\
\text { diagnosis }\end{array}$ & Intervention(s) \\
\hline \multicolumn{5}{|c|}{ Injection studies } \\
\hline 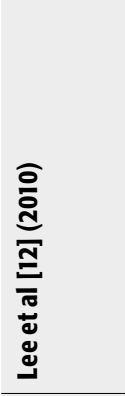 & $\begin{array}{l}\text { BT: } n=20 \\
\text { Age: } 45.1 \pm 13.6 \text { y } \\
\text { Male: } 15 \% \text {, unilateral }(n=20) \\
\text { Duration of pain: } 11.9 \pm 9.1 \mathrm{mo} \\
\text { Injection: } n=19 \\
\text { Age: } 43.2 \pm 12.7 \text { y } \\
\text { Male: } 31.6 \% \text {, unilateral }(n=19) \\
\text { Duration of pain: } 11.9 \pm 14.2 \mathrm{mo} \text {. } \\
\text { FU: BT: } 1,2,3 \text { mo }(85 \%) \\
\text { Injection: } 1 \mathrm{mo}(84.2 \%) \\
2,3 \text { mo }(78.9 \%)\end{array}$ & SIJ syndrome & $\begin{array}{l}\text { Image intensifier-guided single periarticular } \\
\text { injection of lidocaine with } \geq 50 \% \text { pain relief } \\
\text { (measured by NRS) within } 30 \text { min after injection } \\
\text { Additional evaluations: } \\
\text { - Clinical: provocation tests (distraction, } \\
\text { compression, thigh thrust, Patrick and Gaenslen } \\
\text { tests) }\end{array}$ & $\begin{array}{l}\text { Botulinum toxin: } \\
\text { Botulinum type A } \\
\text { Steroid injection: } \\
\text { triamcinolone + lidocaine }\end{array}$ \\
\hline 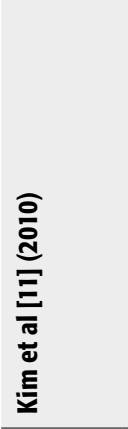 & $\begin{array}{l}\text { Prolotherapy: } n=23 \text { ( } 31 \text { joints) } \\
\text { Age: } 58.7 \pm 13.0 \text { y } \\
\text { Male: } 30 \% \text {, unilateral }(n=15) \\
\text { Bilateral }(n=8) \\
\text { Duration of symptoms: } 40.1(4-240) \text { mo } \\
\text { Injection: } n=25 \text { (35 joints) } \\
\text { Age: } 61.6 \pm 15.2 \text { y } \\
\text { Male: } 28 \% \text {, unilateral }(n=15) \\
\text { Bilateral ( } n=10) \\
\text { Duration of symptoms: } 44.0(3-240) \text { mo } \\
\text { FU: prolotherapy: } 15 \mathrm{mo}(95.8 \%) \\
\text { Injection: } 15 \text { mo }(96.2 \%)\end{array}$ & $\begin{array}{l}\text { History of pain lasting } \geq \\
2 \text { mo in buttock, groin, } \\
\text { or thigh, regardless of } \\
\text { associated lower } \\
\text { extremity measures }\end{array}$ & $\begin{array}{l}\text { Image intensifier-guided single intraarticular } \\
\text { injection of levobupivacaine with a decrease in } \\
\text { pain intensity of } \geq 50 \% \text {, measured by NRS } \\
\text { Additional evaluations: } \\
\text { - Clinical: Patrick test, Gaenslen test, tenderness } \\
\text { over area just below posterosuperior iliac spine }\end{array}$ & $\begin{array}{l}\text { Prolotherapy: dextrose }+ \\
\text { levobupivacaine, mean } 2.7 \\
\pm 1.1 \text { injections } \\
\text { Steroid injection: } \\
\text { triamcinolone }+ \\
\text { levobupivacaine, mean } 1.5 \\
\pm 0.8 \text { injections }\end{array}$ \\
\hline 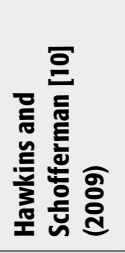 & $\begin{array}{l}N=155 \text { (No. joints NR) } \\
\text { Age: } 48 \text { y, male: 39\%, Unilateral: NR; bilateral: } \\
\text { NR } \\
\text { Duration of symptoms: NR (had received SIJ } \\
\text { injection when history suggestive of SIJ pain) } \\
\text { FU: mean } 44 \text { (26-101) mo (98\%) }\end{array}$ & $\begin{array}{l}\text { All patients in single } \\
\text { spine center who had } \\
\text { received SIJ injection } \\
\text { Previous spine surgery: } \\
\text { - Lumbar, } \mathrm{n}=69\end{array}$ & $\begin{array}{l}\text { Image intensifier-guided injection (to firm } \\
\text { endpoint or leakage) of local anesthetic and } \\
\text { dexamethasone or betamethasone with } \geq 50 \% \\
\text { reduction of pain } 1 \mathrm{~h} \text { after injection and } \\
\text { continuing for } \geq 2 \text { wk } \\
120 / 155 \text { (77\%) experienced pain relief, } 118 \text { of } \\
\text { them received therapeutic injections }\end{array}$ & $\begin{array}{l}\text { Local anesthetic }+ \\
\text { dexamethasone or } \\
\text { betamethasone, mean } 2.7 \\
(1-9) \text { injections }\end{array}$ \\
\hline 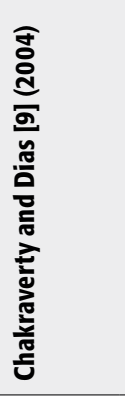 & $\begin{array}{l}\text { Injection: } \mathrm{n}=33 \\
\text { Age: } 40(17-74) \text { y } \\
\text { Male: } 39.4 \% \\
\text { Duration of pain: } 4(0.5-10) \text { y } \\
\text { Prolotherapy: } n=19 \\
\text { Age: } 41 \text { ( } 17-58) \text { y } \\
\text { Male: } 31.6 \% \\
\text { Duration of pain: } 6 \text { y }(2-15) \\
\text { FU: injection: } \leq 6 \text { mo }(97 \%) \\
\text { Prolotherapy: } 3 \text { mo }(100 \%) \\
6,12 \text { mos }(94.7 \%)\end{array}$ & $\begin{array}{l}\text { Chronic LBP patients } \\
\text { with presumed SIJ or } \\
\text { facet joint pain }\end{array}$ & $\begin{array}{l}\text { Image intensifier-guided, contrast confirmed, } \\
\text { single intraarticular SIJ injection of lignocaine/ } \\
\text { triamcinolone with } \geq 50 \% \text { pain relief (based on } \\
\text { VAS score) within the first hour after injection } \\
33 / 52(63.5 \%) \text { experienced pain relief and } \\
\text { received therapeutic corticosteroid injection } \\
19 / 52(36.5 \%) \text { experienced no pain relief and } \\
\text { received therapeutic prolotherapy }\end{array}$ & $\begin{array}{l}\text { Injection: SIJ intraarticular } \\
\text { corticosteroid injection } \\
\text { Prolotherapy: ipsilateral } \\
\text { iliolumbar and SI } \\
\text { interosseus ligament } \\
\text { injection of dextrose or } \\
\text { P2G + lignocaine, } \\
3 \text { injections }\end{array}$ \\
\hline 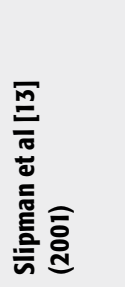 & $\begin{array}{l}\mathrm{N}=31 \text { (No. of joints NR) } \\
\text { Age: } 44.8(22-80) \text { y } \\
\text { Male: } 38.7 \% \text {, unilateral NR } \\
\text { Bilateral NR } \\
\text { Duration of pain: mean } 20.6(1.5-84) \text { mo } \\
\text { FU: Mean } 94.4(10-60) \text { wk }(82.9 \%)\end{array}$ & $\begin{array}{l}\text { LBP including sacral } \\
\text { sulcus region regardless } \\
\text { of associated hip or leg } \\
\text { symptoms }\end{array}$ & $\begin{array}{l}\text { Image intensifier-guided single SIJ injection of } \\
\text { lidocaine with } \geq 80 \% \text { reduction of pain within } 30 \\
\text { min using VAS scale } \\
\text { Additional evaluations: } \\
\text { - Clinical: positive response to } \geq 3 \text { of the } \\
\text { following: Patrick and Gaenslen test, pain with } \\
\text { pressure applied to SI ligaments, shear test, } \\
\text { Yeoman maneuver }\end{array}$ & $\begin{array}{l}\text { Intraarticular injection of } \\
\text { betamethasone and } \\
\text { lidocaine, mean } 2.14(1-4)\end{array}$ \\
\hline
\end{tabular}

* See full table in the Web Appendix. CoE indicates class of evidence; CT, computed tomography; MRI, magnetic resonance imaging; NR, not reported; FU, follow-up; SIJ, sacroiliac joint; OA, osteoarthritis; IA, inflammatory arthritis; LBP, low back pain; P2G, phenol, glycerine, glucose; BT, Botulinum toxin type A; NRS, numeric rating scale for pain; MMPI, Minnesota Multiphasic Personality Inventory; FPI, Freiburg personality inventory; FAPK, questionnaire for recognition of psychosomatic diseases; ICBG, iliac crest bone graft. Although these studies were designed to compare injection treatments, for the purpose of this review they are considered case series, with each arm addressed separately, since the study question focuses on comparing surgery with injection $[9,11,12]$

† Demographics applicable to patients analyzed [1 1]; patients completing all discharge questionnaires [13]; and patients before diagnostic block performed ( $\mathrm{n}=118$ patients had positive diagnostic block and were included in study) [10]. Study also included 42 patients who received a lumbar facet joint injection and 10 patients who received a medial branch block [9]. 


\section{Pain (Fig 2 and Web Appendix)}

- All eight studies evaluating pain reported improvement regardless of the treatment, with most studies reporting over $40 \%$ improvement in pain as measured by VAS or NRS score [2, 4, 11-13]. Two studies described minimal clinically important differences (MCID) [2, 12]. Based on indirect comparison of surgical and injection studies, improvement from baseline pain was similar for both treatment options.

- Four of the seven surgical studies [4] reported on pain, with debridement patients showing greater pain improvement than fusion patients [2]. One study [6] reported only 3 patients (17.6\%) experiencing mild/no pain compared with 14 patients $(82.4 \%)$ experiencing marked/severe pain at 39 months.
- Of four injection studies reporting on pain, most patients receiving Botulinum toxin injection [12] or prolotherapy [11] experienced greater pain relief than patients receiving corticosteroid therapy [11-13]. One study [10] found that $78(66.1 \%)$ of 118 patients required two or more injections to achieve at least $50 \%$ pain relief for 2 or more weeks.

- Al-Khayer et al [2] defined the MCID for VAS as a 2 -point score change, with $9(100 \%)$ of 9 fusion patients achieving the MCID. Another study [12] defined the MCID for NRS as a $50 \%$ reduction in score, reporting that $88 \%$ of patients receiving Botulinum toxin injection exceeded the MCID.

Fig 2 Percentage improvement from baseline in VAS (Visual Analog Score) or NRS (Numeric Rating Score) at follow-up in patients who underwent surgical or injection treatment for chronic sacroiliac joint pain.

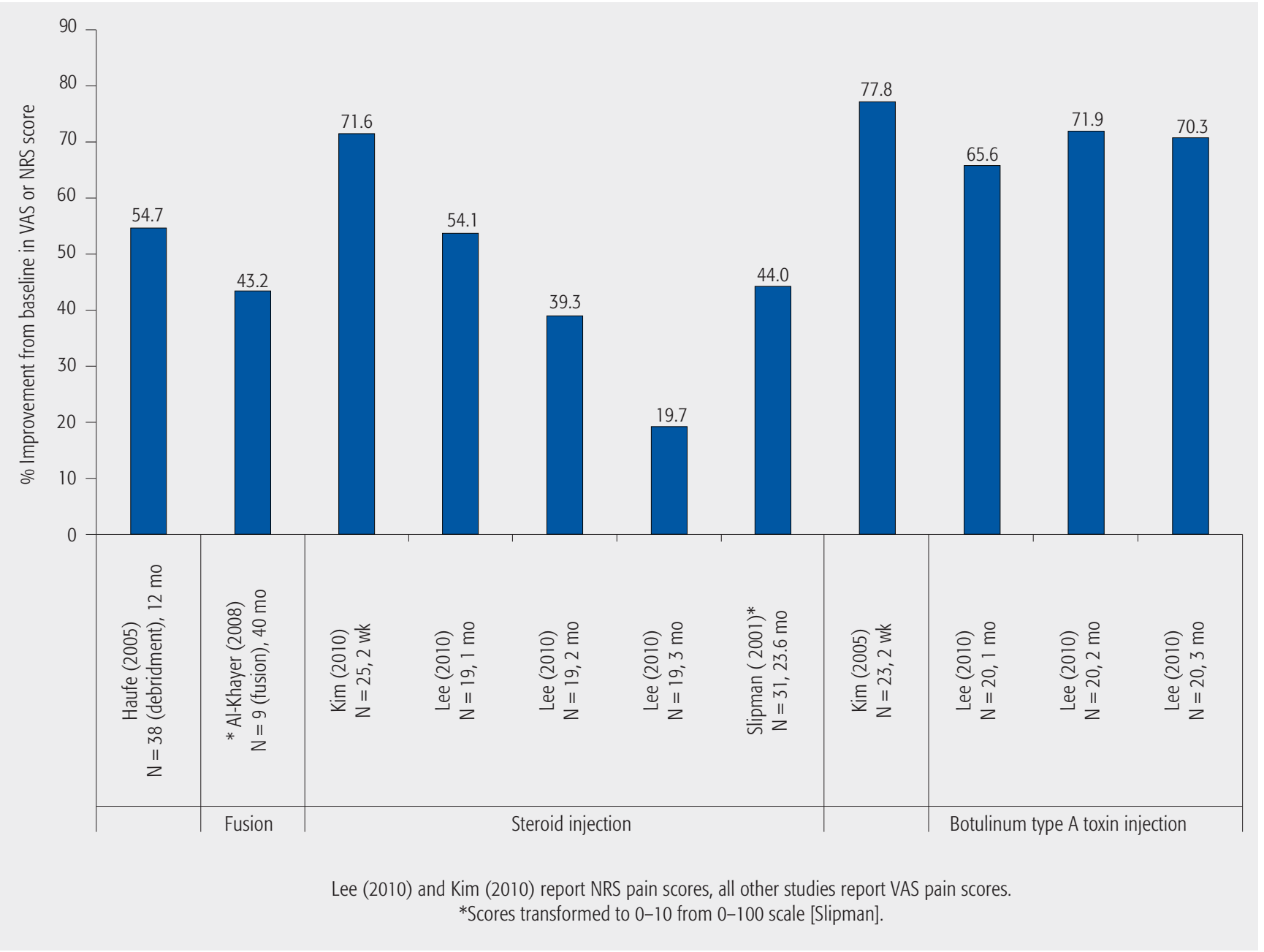




\section{Functional and quality-of-life outcomes (Table 2 and Fig 3 )}

- The four surgical and two injection studies evaluating functional outcomes reported improvement as measured by ODI, AAOS/MODEMS, or the Majeed score regardless of the treatment, with most studies reporting over $20 \%$ improvement in functionality. Conclusions regarding the comparative effectiveness of surgical treatment versus injection are not possible.

- One debridement study [4] reported that 23 (60\%) of 38 patients experienced an improved ODI score, with only $1(2 \%)$ of 38 experiencing increased impairment at over 2 years' follow-up (details NR).

- In several injection studies, patients receiving a Botulinum toxin injection or prolotherapy experienced over
$50 \%$ improvement in ODI scores [11, 12]. Studies using corticosteroid injections exhibited inconsistent results [11-13].

- Al-Khayer et al [2] defined the MCID for ODI as a $10 \%$ change, with $8(88.9 \%)$ of 9 fusion patients achieving MCID. Another study defined the MCID for ODI as a $40 \%$ reduction in score and reported that $88 \%$ of patients receiving Botulinum toxin injection exceeded the MCID [12].

- Two surgical fusion studies evaluating health-related quality of life reported improvement measured by the SF-36 [3, 5]. Results for mean improvement in SF-36 Physical Component Summary were inconsistent.

Fig 3 Percentage improvement from baseline in ODI (Oswestry Disability Index) at follow-up in patients who underwent surgical or injection treatment for chronic sacroiliac joint pain.

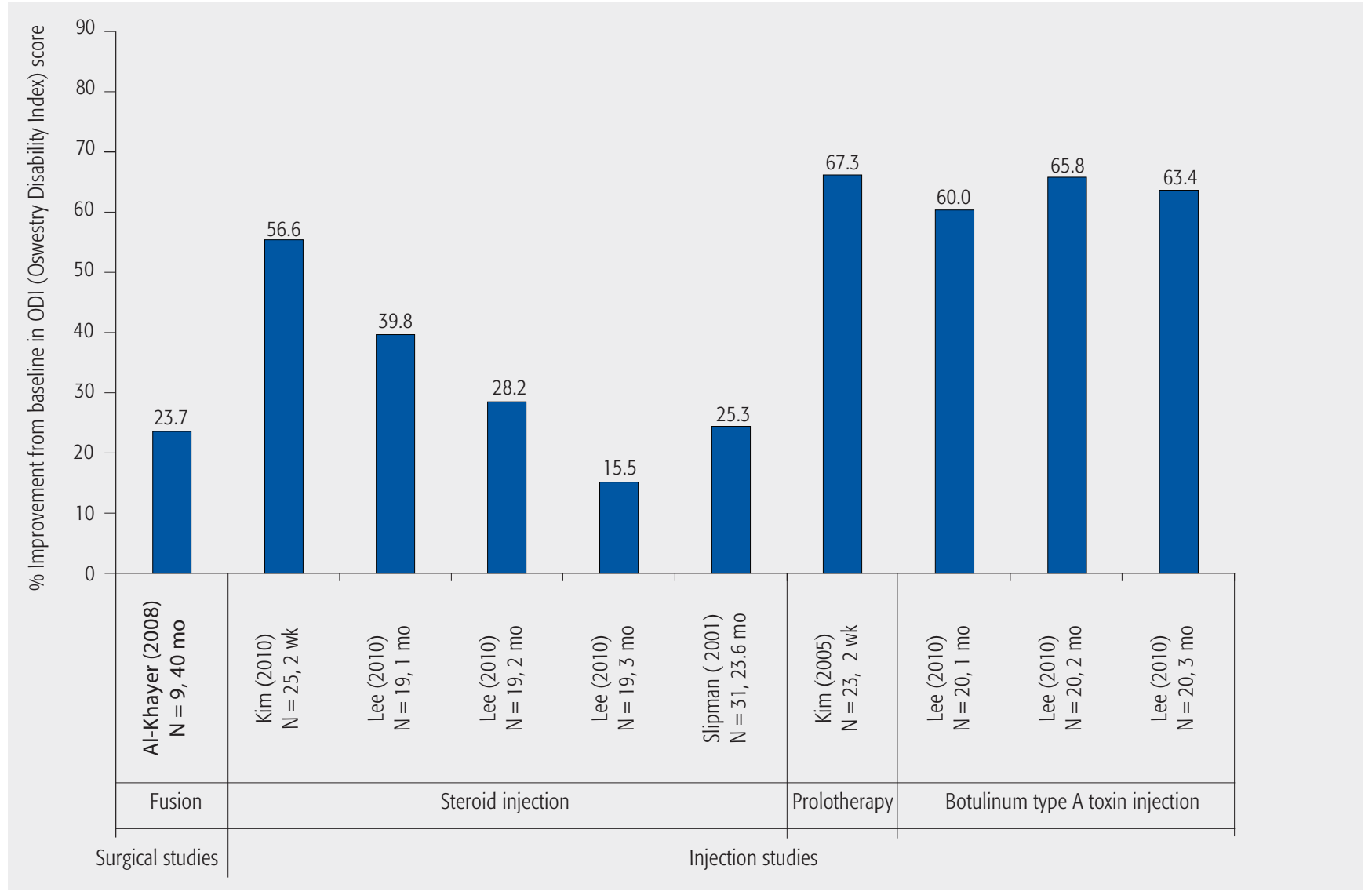


Table 2 Functional outcome (Majeed and AAOS/MODEM) scores and health-related quality of life (SF-36) outcome scores from baseline to follow-up in surgical studies on treatment for chronic sacroiliac joint pain.*

\begin{tabular}{|c|c|c|c|c|c|}
\hline & Scoring system (component) & $\begin{array}{l}\text { Preoperative } \\
\text { score, mean } \pm \text { SD }\end{array}$ & Follow-up & $\begin{array}{l}\text { Score at follow-up, } \\
\text { mean } \pm S D \text { (range) }\end{array}$ & $\begin{array}{l}\text { Improvement } \\
\text { from baseline, } \%\end{array}$ \\
\hline \multicolumn{6}{|l|}{ Functional outcomes } \\
\hline Khurana et al [5] (2009) & Majeed score & $37(18-54)$ & $17 \mathrm{mo}$ & $79(62-96)$ & 113.5 \\
\hline \multirow[t]{2}{*}{ Buchowski et al [3] (2005) } & $\begin{array}{l}\text { AAOS/MODEMS Satisfaction with } \\
\text { symptoms index }\end{array}$ & $1.20 \pm 0.77$ & $5.8 y$ & $2.67 \pm 1.63$ & 122.5 \\
\hline & AAOS/MODEMS Pain/disability index & $34.91 \pm 12.39$ & $5.8 \mathrm{y}$ & $57.45 \pm 23.04$ & 64.6 \\
\hline \multicolumn{6}{|c|}{ Health-related quality-of-life outcome } \\
\hline \multirow[t]{2}{*}{ Khurana et al [5] (2009) } & SF-36 (Physical Component Summary) & $28.49 \pm 11.24$ & $17 \mathrm{mo}$ & $51.38 \pm 9.87$ & 80.3 \\
\hline & SF-36 (Mental Component Summary) & $46.82 \pm 17.88$ & $17 \mathrm{mo}$ & $57.48 \pm 17.32$ & 22.8 \\
\hline \multirow[t]{2}{*}{ Buchowski et al [3] (2005) } & SF-36 (Physical Component Summary) & $26.37 \pm 7.35$ & $5.8 \mathrm{y}$ & $33.53 \pm 11.72$ & 27.2 \\
\hline & SF-36 (Mental Component Summary) & $42.45 \pm 12.08$ & $5.8 y$ & $49.46 \pm 13.07$ & 16.5 \\
\hline
\end{tabular}

* Majeed scoring system was developed to assess functional outcome after pelvic injuries and is based on five criteria: pain (30 patients), standing (36 patients), sitting (10 patients), sexual function (4 patients), performance at work (20 patients) (0-00 points; lower score indicates worse functionality) [Khurana].

AAOS/MODEMS (American Academy/Association of Orthopaedic Surgeons/Musculoskeletal Outcomes Data Evaluation and Management System) is a modified Oswestry Disability Index scale and includes seven sections; lower score indicates greater disability [Buchowski, Longo]. SF-36 (Short-Form 36) measures quality of life in multiple domains, each domain with a maximum of 100 points (lower scores indicate greater disability); the Physical Component Summary is an aggregate of the Physical Functioning, Role-Physical, Bodily Pain, and General Health scales; the Mental Component Summary is an aggregate of the Vitality, Social Functioning, Role-Emotional, and Mental Health scales [Buchowski, Khurana]. Transformed scores reported; transformation not defined [Buchowski].

\section{Fusion (surgical studies only)}

- Four studies reported fusion rates ranging from $85 \%-100 \%$ at follow-ups covering 6-40 months [2, $3,5,8]$ and one fusion study [6] reported that $58.8 \%$ of patients experienced definite/questionable fusion. Fusion was determined by radiographs alone in two studies [2, 3], mixed radiographs, and CT scans in one study [6], radiographs for all and additional CT scans on symptomatic patients in one study [5], and CT scans for all patients in one study [8]. Scant details were provided in a few studies regarding the criteria for determining successful fusion, including absence of clinical symptoms and absence of radiological signs of metal failure or lucency [5], bone fusion of sacroiliac joint visible on CT scan [6], and absence of loosening around cages and evidence of bone bridging through the cage from the sacrum to the ileum [8].

\section{Return to work}

- One fusion study reported that $4(44.4 \%)$ of 9 patients had returned to work at 40-month follow-up [2], with another fusion study reporting $8(40 \%)$ of 20 patients returning to work at the 5.8-year follow-up (mean: $5.0 \pm 1.6$ month-time to return to work) [3].

- One injection study reported that no patients experienced a worsened work status, $13(54.2 \%)$ of 24 reported an unchanged work status, and $11(45.8 \%)$ of 24 reported an improved work status at a mean of 23.6-month follow-up [13].

\section{Complications}

- Five surgical studies reported complications $[2,3$, 6-8]. Infection rates varied from $4.8 \%-11.1 \%$ (mean follow-up of 30 months to 5.8 years) $[2,3,7]$. Nonunion occurred in $7.7 \%-41.2 \%$ of patients in four surgical studies (mean follow-up of 29.5 months to 5.8 years) [3, 6-8]. Further surgery was reported in two surgical studies for hardware removal, scar tissue excision, and a second fusion: $1(5 \%)$ of 20 patients (5.8-year follow-up) [3] and $11(64.7 \%)$ of 17 patients (39-month follow-up) [6]). An intraoperative fracture was reported in $1(5.9 \%)$ of 17 patients [6].

- One injection study reported flu-like symptoms in 2 $(10 \%)$ of 20 patients receiving Botulinum toxin [12]. 


\section{CLINICAL GUIDELINES}

- Numerous guidelines were found addressing sacroiliac joint pain or low back pain; however, few address the use of surgery for relief of CSJP:

- The Work Loss Data Institute recommended sacroiliac joint block/injections[14, 15], has sacroiliac joint debridement under study [14], and did not recommend sacroiliac joint fusion or radiofrequency neurotomy [14] for work-related injuries [14] and work-related low back pain [15].

- A practice guideline for chronic pain management recommended sacroiliac joint injections for symptomatic relief of sacroiliac joint pain [16].
- An occupational medicine practice guideline recommended sacroiliac joint corticosteroid injections for acute, low back pain, known sacroiliitis, spinal fractures, radicular pain syndrome, and low back pain believed to be due to the sacroiliac joint [17].

- A European Cooperation in Science and Technology (COST) guideline for the management of chronic nonspecific low back pain [18] did not recommend sacroiliac joint corticosteroid injections due to limited evidence.

- The American Society of Interventional Pain Physicians' guidelines [19] for the management of chronic spinal pain found limited evidence for the use of intraarticular sacroiliac joint injections in short-term and long-term sacroiliac joint pain relief and described moderate evidence for sacroiliac joint blocks to diagnose sacroiliac joint pain.

\section{EVIDENCE SUMMARY (Table 3)}

The overall strength of evidence is very low for outcomes common to the two treatments (surgical and injection) and is based on lack of comparative studies and small sample sizes. Determination of effect sizes for differences between surgical and injection treatment is not possible and further comparative research is needed.

Table 3 Compare pain, functionality, quality of life, and return to work for surgical vs injection treatment of injection-confirmed sacroiliac joint pain.

\begin{tabular}{|c|c|c|c|c|c|}
\hline Outcomes & \multicolumn{4}{|c|}{ Strength of evidence } & \multirow{2}{*}{$\begin{array}{l}\text { Conclusions/comments } \\
\text { Regardless of the type of treatment, most studies reported over 40\% } \\
\text { improvement in pain as measured by VAS or NRS score }\end{array}$} \\
\hline 1. Improvement in pain & Very low & Low & Moderate & High & \\
\hline $\begin{array}{l}\text { 2. Improvement in } \\
\text { functionality }\end{array}$ & Very low & Low & Moderate & High & $\begin{array}{l}\text { Regardless of the type of treatment, most studies reported over 20\% } \\
\text { improvement in functionality }\end{array}$ \\
\hline 3. Return to work & Very low & Low & Moderate & High & $\begin{array}{l}\text { Two fusion and one injection study reported over } 40 \% \text { of patients } \\
\text { returning to work at follow-up }\end{array}$ \\
\hline \multicolumn{6}{|c|}{ Compare complications for surgical vs injection treatment of injection-confirmed sacroiliac joint pain } \\
\hline Outcomes & \multicolumn{4}{|c|}{ Strength of evidence } & Conclusions/comments \\
\hline 1. Treatment related & Very low & Low & Moderate & $\stackrel{\text { High }}{\text { High }}$ & $\begin{array}{l}\text { Most complications were reported in surgical studies, including } \\
\text { infections, nonunion, further surgery, and intraoperative fracture. } \\
\text { Only one injection study reported complications (flu-like symptoms } \\
\text { in the Botulinum toxin arm of the study) }\end{array}$ \\
\hline
\end{tabular}




\section{ILLUSTRATIVE CASE}

The patient is a 37-year-old retired professional cyclist with 8 years' status post L4-S1 fusion for recurrent disc herniations, and 3 years' status post L3-4 fusion for adjacent segment degeneration (Figs 4 and $\mathbf{5}$ ). Each of these surgeries was successful in relieving her preoperative pain. One year after L3-4 interbody fusion, she began experiencing increasing left, low back and buttock pain consistent on examination with pain emanating from her sacroiliac joint. Her symptoms were initially relieved with physical therapy directed at her left sacroiliac joint, and later a sacroiliac joint belt, which helped for a brief period. With recurrent symptoms, she underwent a series of guided sacroiliac corticosteroid injections that each significantly relieved her pain. Eventually, she failed to respond to further injections, therapy, or oral medications and elected to undergo a mini-open sacroiliac joint fusion (Fig 6), with placement of percutaneous sacroiliac screws and an iliac bolt connected to her posterior instrumentation (Figs 7 and $\mathbf{8}$ ). Postoperatively, she noted immediate and remarkable pain relief, and 1 year after sacroiliac joint fusion she is virtually pain free and has returned to full and unrestricted activity.
Fig 4 AP lumbosacral $x$-rays s/p staged L3-S1 fusion. Moderate degenerative sacroiliac joint changes noted on the right.

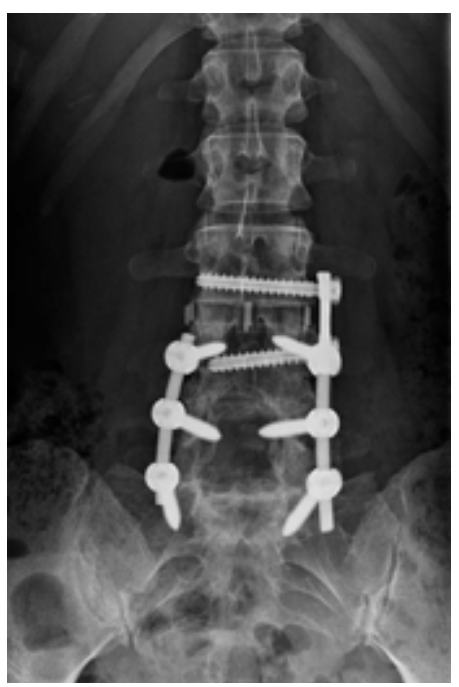

Fig 5 Lateral lumbosacral x-rays s/p staged L3-S1 fusion. Moderate degenerative sacroiliac joint changes noted on the right.

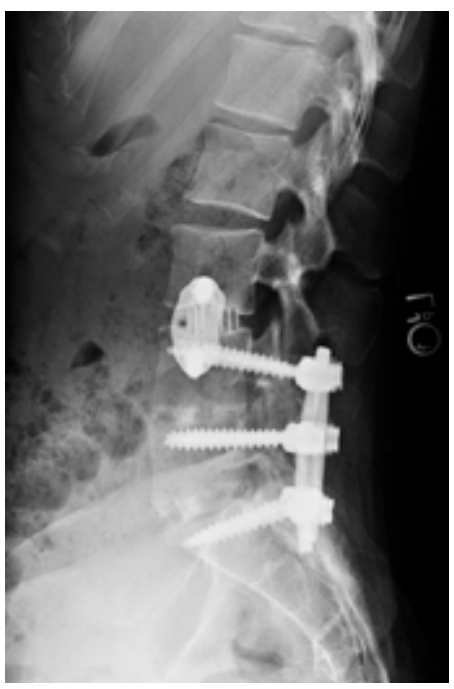

Fig 6 Intraoperative axial view of right sacroiliac joint after removal of medial half of the posterosuperior iliac spine and debridement of the joint to within 1 inch of anterior cortex.

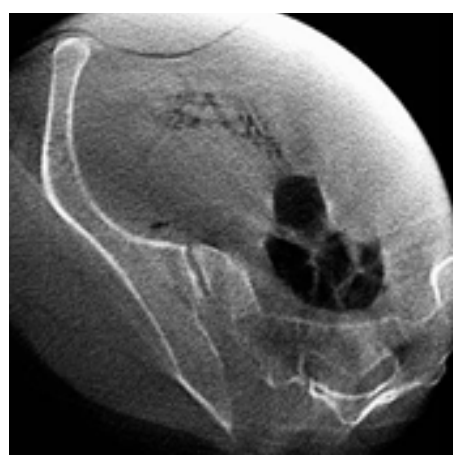


Fig 7 AP x-rays s/p open right sacroiliac joint fusion with iliac bolt attached to previous instrumentation and percutaneous sacroiliac screw placement.

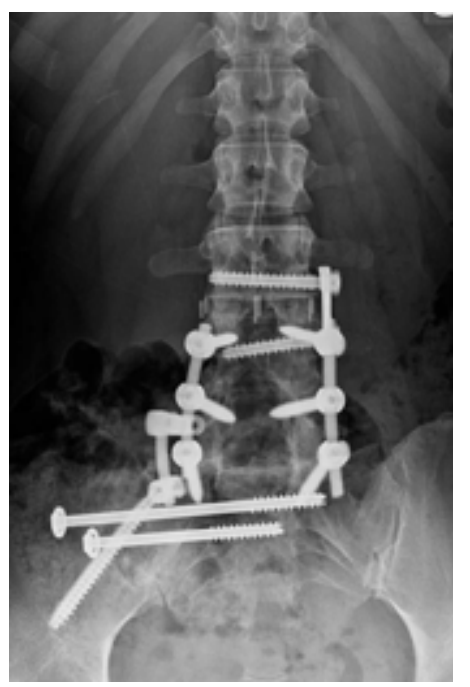

Fig 8 Lateral $x$-rays s/p open right sacroiliac joint fusion with iliac bolt attached to previous instrumentation and percutaneous sacroiliac screw placement.

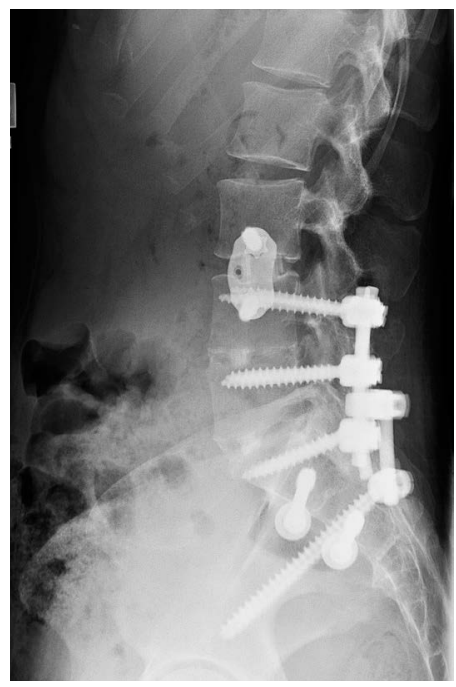

\section{DISCUSSION}

- This systematic review is limited by the following:

- The existing literature reporting outcomes on surgical treatment of CSJP is limited to case series. No studies were identified that compared surgical with injection treatment in the same patient population, so conclusions regarding the comparative effectiveness of the treatments are not possible.

- Most studies comprised small sample sizes and reported short follow-up time, bringing into question the duration of treatment effect. The study population included a mixture of diagnoses and many patients who had undergone previous spinal surgery, making direct comparisons of the interventions difficult to interpret.

- There was heterogeneity with respect to procedural details in the injection studies, including the type of injectant and number of injections. These differences must be considered when interpreting the findings of this systematic review.

- Fusion results should be interpreted with caution, as two of the five studies that provide the basis for the fusion results used plain radiographs only (of limited value in confirming fusion), while only a single study used CT scans in all patients to determine fusion.

- All studies reported pain relief regardless of the intervention. The amount and duration of pain relief was highly variable and difficult to compare.

- Complication and infection rates are likely higher with surgical intervention than with therapeutic injections as suggested by this review.

- The data suggests similar outcomes in patients treated with surgical fusion and those treated with therapeutic injections, with increased complications reported in surgical patients. Thus, surgical intervention should likely be reserved for patients who have failed nonoperative treatment modalities.

- There is a need for a well-designed comparative study to evaluate the effectiveness of the current treatment modalities used for CSJP. 


\section{REFERENCES}

1. Ashman B, Norvell DC, Hermsmeyer JT (2010) Chronic sacroilac joint pain: fusion versus denervation as treatment options. EBSJ; 1(3):35-44.

2. Al-Khayer A, Hegarty J, Hahn D, et al (2008) Percutaneous sacroiliac joint arthrodesis: a novel technique. J Spinal Disord Tech; 21(5):359-363.

3. Buchowski JM, Kebaish KM, Sinkov V, et al (2005) Functional and radiographic outcome of sacroiliac arthrodesis for the disorders of the sacroiliac joint. Spine J; 5(5):520-528; discussion 529.

4. Haufe SM, Mork AR (2005) Sacroiliac joint debridement: a novel technique for the treatment of sacroiliac joint pain. Photomed Laser Surg; 23(6):596-598.

5. Khurana A, Guha AR, Mohanty K, et al (2009) Percutaneous fusion of the sacroiliac joint with hollow modular anchorage screws: clinical and radiological outcome. J Bone Joint Surg Br; 91(5):627-631.

6. Schutz U, Grob D (2006) Poor outcome following bilateral sacroiliac joint fusion for degenerative sacroiliac joint syndrome. Acta Orthop Belg; 72(3):296-308.

7. Waisbrod H, Krainick JU, Gerbershagen HU (1987) Sacroiliac joint arthrodesis for chronic lower back pain. Arch Orthop Trauma Surg; 106(4):238-240.

8. Wise CL, Dall BE (2008) Minimally invasive sacroiliac arthrodesis: outcomes of a new technique. J Spinal Disord Tech; 21(8):579-584.

9. Chakraverty R, Dias R (2004) Audit of conservative management of chronic low back pain in a secondary care setting-part I: facet joint and sacroiliac joint interventions. Acupunct Med; 2(4):207-213.

10. Hawkins J, Schofferman J (2009) Serial therapeutic sacroiliac joint injections: a practice audit. Pain Med; 10(5):850-853.

11. Kim WM, Lee HG, Jeong CW, et al (2010) A randomized controlled trial of intra-articular prolotherapy versus steroid injection for sacroiliac joint pain. J Altern Complement Med; 16(12):1285-1290.

12. Lee JH, Lee SH, Song SH (2010) Clinical effectiveness of botulinum toxin A compared to a mixture of steroid and local anesthetics as a treatment for sacroiliac joint pain. Pain Med; 11(5):692-700.
13. Slipman CW, Lipetz JS, Plastaras CT, et al (2001) Fluoroscopically guided therapeutic sacroiliac joint injections for sacroiliac joint syndrome. Am J Phys Med Rehabil; 80(6):425432.

14. Work Loss Data Institute (2011) Hip and pelvis (acute and chronic). Available at: www. guideline.gov

15. Work Loss Data Institute (2011) Low back: lumbar and thoracic (acute and chronic). Available at: www.guideline.gov

16. American Society of Anesthesiologists Task Force on Chronic Pain Management, American Society of Regional Anesthesia and Pain Medicine (2010) Practice guidelines for chronic pain management: an updated report by the American Society of Anesthesiologists Task Force on Chronic Pain Management and the American Society of Regional Anesthesia and Pain Medicine. Anesthesiology; 112(4):810833.

17. American College of Occupational and Environmental Medicine Practice Guidelines Committee (2007) Low back disorders: occupational medicine practice guidelines: evaluation and management of common health problems and functional recovery in workers. Elk Grove Village, IL: American College of Occupational and Environmental Medicine (ACOEM).

18. Airaksinen O, Brox Jl, Cedraschi C, et al (2006) European guidelines for the management of chronic nonspecific low back pain. Eur Spine J; 15 Suppl 2:S192-300.

19. Boswell MV, Trescot AM, Datta S, et al (2007) Interventional techniques: evidencebased practice guidelines in the management of chronic spinal pain. Pain Physician; 10(1): 7-111. 


\section{EDITORIAL PERSPECTIVE}

The reviewers welcomed the thoughtfulness and care of Spiker et al in this study - the most exhaustive assessment of comparative effectiveness of sacroiliac (SI) injection to surgery to date. Going back to the article by Ashman et al [1] in EBSJ in which denervation and fusion procedures were compared, this topic has remained confounding and elusive. The very diagnosis of SI pain as a distinct somatic entity remains unclear as its origin does not seem to be clearly diagnosable [2, 3]. As there remains a great deal of uncertainty about diagnosing the source of pain in patients with pain in their SI area, actual treatment will remain necessarily vague as well. On the treatment side, many questions remain:

1. Was the cause of SI joint pain ever really sub-differentiated in terms of treatment response? For instance, was an attempt made to differentiate SI joint pain into various entities, such as primary instability, degenerative arthritis, and mechanical overload in presence of long lumbosacral fusion, lumbosacral malalignment, posttraumatic conditions, and inflammatory arthropathies? Also, age and SI joint arthropathy play an important role. How did age factor into the onset of patient complaints? It would seem helpful to create some subgroups as not all will respond the same to any treatment.

2. What convinces the authors that their SI injections are definitely intraarticular? The SI joint is a pleomorphic articulation, usually C-shaped, but sometimes it actually consists of two separate articular surfaces on either side. The gold standard for an SI joint injection would seem to be a CTguided and confirmed injection and not an injection with conventional 2-D imaging, even if it is done with arthrography. What was actually injected, local anesthetic plus steroids or in isolation, and what specific variant of either medication was used? Again, there seems to be substantial practice variations clouding any study attempt.

3. Quality of fusion: As previously identified in our commentary on the Ashman and coworkers' article [1], the actual presence of a fusion appears to be assumed rather than confirmed with imaging, such as CT scans, thus again inserting a potential for systematic observer error.
As reported by Spiker et al, perhaps the most disturbing aspect of the presented surgical studies are the high-reported complication rates with up to $44.5 \%$ nonunion rates. These procedures are clearly not benign but require a thoughtful, sustained nonoperative treatment approach before engaging in surgical attempts, and if these are undertaken, experience in this area may be desirable.

The many unanswered questions regarding SI joints would seemingly invite a prospective trial with sham injections, for instance, to prove value of injection therapy, or a comparison trial with various types of SI joint fusion. None of this has occurred to date, but hopefully readers of this article will feel compelled to fill in the gaps in this area.

1. Ashman B, Norvell DC, Hermsmeyer JT (2010) Chronic sacroilac joint pain: fusion versus denervation as treatment options. EBSJ; 1(3):35-44.

2. Dreyfuss $\mathbf{P}$, Michaelsen $\mathbf{M}$, Pauza K, et al (1996) The value of medical history and physical examination in diagnosing sacroiliac joint pain. Spine (Phila Pa 1976); 21(22):2594-2602.

3. Dreyfuss $P$, Dreyer SJ, Cole $A$, et al (2004) Sacroiliac joint pain. J Am Acad Orthop Surg; 12(4):255-265. 